\title{
Volume Assessment in Mechanically Ventilated Critical Care Patients Using Bioimpedance Vectorial Analysis, Brain Natriuretic Peptide, and Central Venous Pressure
}

\author{
Andrew A. House, ${ }^{1}$ Mikko Haapio, ${ }^{2}$ Paolo Lentini, ${ }^{3}$ Ilona Bobek, ${ }^{3}$ Massimo de Cal, ${ }^{4}$ \\ Dinna N. Cruz, ${ }^{3}$ Grazia M. Virzì, ${ }^{3}$ Rizzieri Carraro, ${ }^{5}$ Giampiero Gallo, ${ }^{5}$ Pasquale Piccinni, ${ }^{5}$ \\ and Claudio Ronco ${ }^{3}$
}

${ }^{1}$ Division of Nephrology, London Health Sciences Center, London, Canada N6A 5A5

${ }^{2}$ Helsinki University Central Hospital, Division of Nephrology, 00029 Helsinki, Finland

${ }^{3}$ St Bortolo Hospital, Department of Nephrology, 36100 Vicenza, Italy

${ }^{4}$ Division of Nephrology, Department of Medical and Surgical Sciences, University of Padua, 35128 Padua, Italy

${ }^{5}$ St Bortolo Hospital, Department of Anesthesia and Intensive Care, 36100 Vicenza, Italy

Correspondence should be addressed to Andrew A. House, andrew.house@lhsc.on.ca

Received 6 July 2010; Accepted 28 September 2010

Academic Editor: Mitchell H. Rosner

Copyright ( 2011 Andrew A. House et al. This is an open access article distributed under the Creative Commons Attribution License, which permits unrestricted use, distribution, and reproduction in any medium, provided the original work is properly cited.

Purpose. Strategies for volume assessment of critically ill patients are limited, yet early goal-directed therapy improves outcomes. Central venous pressure (CVP), Bioimpedance Vectorial Analysis (BIVA), and brain natriuretic peptide (BNP) are potentially useful tools. We studied the utility of these measures, alone and in combination, to predict changing oxygenation. Methods. Thirty-four mechanically ventilated patients, 26 of whom had data beyond the first study day, were studied. Relationships were assessed between CVP, BIVA, BNP, and oxygenation index $\left(\mathrm{O}_{2} \mathrm{I}\right)$ in a cross-sectional (baseline) and longitudinal fashion using both univariate and multivariable modeling. Results. At baseline, CVP and $\mathrm{O}_{2} \mathrm{I}$ were positively correlated $(R=0.39 ; P=.021)$, while CVP and BIVA were weakly correlated $(R=-0.38 ; P=.025)$. The association between slopes of variables over time was negligible, with the exception of $\mathrm{BNP}$, whose slope was correlated with $\mathrm{O}_{2} \mathrm{I}(R=0.40 ; P=.044)$. Comparing tertiles of CVP, $\mathrm{BIVA}$, and BNP slopes with the slope of $\mathrm{O}_{2} \mathrm{I}$ revealed only modest agreement between $\mathrm{BNP}$ and $\mathrm{O}_{2} \mathrm{I}($ kappa $=0.25 ; P=.067)$. In a regression model, only BNP was significantly associated with $\mathrm{O}_{2} \mathrm{I}$; however, this was strengthened by including CVP in the model. Conclusions. BNP seems to be a valuable noninvasive measure of volume status in critical care and should be assessed in a prospective manner.

\section{Purpose}

Volume assessment and management in critically ill patients remains challenging [1]. Issues of timing, choice, amount of fluids, and type of volume assessments to guide therapy continue to be investigated. While early volume resuscitation and goal-directed therapy have been shown to improve mortality and morbidity $[2,3]$ and lessen the risk of acute kidney injury [3], management of patients with established acute lung injury reveals that a more conservative or "dry" strategy is more appropriate than a liberal or "wet" one
[4]. Studies of the assessment of fluid status have shown that simple central venous pressure (CVP) monitoring is as effective, and safer, than more invasive means such as pulmonary artery occlusion pressure [5]. It is clear, however, that CVP does not tell the entire story, as patients with high right sided pressures may have reduced, normal, or increased effective circulating volume.

Bioimpedance vectorial analysis (BIVA) allows determination of extracellular fluid volume and total body water from measurements of resistivity of tissues to single or multifrequency emitted signals. BIVA has been used to 
manage volume in hemodialysis patients for several decades. However, the use of BIVA in critically ill patients has not been extensively studied, and the data used to determine volume status have been derived from hemodynamically stable patients $[6,7]$.

Brain natriuretic peptide (BNP) is a biomarker used to identify patients with fluid overload and congestive heart failure [8]. In critical care, it has been shown to correlate with mortality and morbidity, though it has not been used to guide therapy $[9,10]$.

We conducted a pilot study to examine the relationships between CVP, BIVA, and BNP in order to determine which measure, or combination of measures, relate to volume status in critically ill, ventilated patients.

\section{Methods}

This study was approved by the Institutional Review Board of the San Bortolo Hospital, Vicenza, Italy, and conducted in the Intensive Care Unit (ICU). Any adult patient requiring mechanical ventilation was eligible. Because of the technical requirements for BIVA, patients with any upper or lower limb amputation, severe rhabdomyolysis, or erysipelas of both upper or lower limb were excluded. As the study required serial measurements over time, any patient not expected to survive 72-96 hours was excluded. Any patient with recent cardiac surgery was also excluded, as BNP and CVP may be grossly skewed. Likewise, patients with decompensated heart failure or acute coronary syndrome were excluded. As published, BIVA vectors were derived in Caucasians [6], we excluded non-Caucasians. A sample size of 30-40 patients was enrolled without formal sample-size calculations.

Within 48-72 hours of initiating mechanical ventilation, baseline assessment was undertaken including CVP, BIVA, and blood sample for BNP, hematocrit, and creatinine. BNP was determined using Triage MeterPro (Biosite Inc., San Diego, CA). CVP and BIVA were recorded in a blinded fashion by separate trained observers. CVP was obtained through a central venous catheter connected to a calibrated transducer using the level of the right atrium as a reference point. BIVA was performed using a plethysmograph emitting $800-\mu \mathrm{A}$ and $50-\mathrm{kHz}$ alternating sinusoidal current (EFG Electrofluidgraph, Akern s.r.l., Pontassieve, Florence, Italy) and previously published methods [11]. Clinical data were recorded, including primary illness, systolic blood pressure (SBP), diastolic blood pressure (DBP), mean arterial pressure (MAP), heart rate (HR), weight, urine output, pressor doses, $\mathrm{PO}_{2}, \mathrm{FiO}_{2}$, and mean airway pressure. Additional measurements were made at 24 and 48 hour intervals to minimize diurnal variation in BNP. The fluid balance in the intervening period was calculated.

CVP was categorized as "low" $\left(<4 \mathrm{~cm} \mathrm{H}_{2} \mathrm{O}\right)$, "high" $\left(\geq 14 \mathrm{~cm} \mathrm{H} \mathrm{H}_{2} \mathrm{O}\right.$ ), or "normal" (4-14 cm $\mathrm{H}_{2} \mathrm{O}$ ) [4]. For the BIVA, three patterns were considered according to published references for resistance/height $(\mathrm{R} / \mathrm{H})$ based on normals, adjusted for age, sex, and weight [7]: long vectors outside the $75 \%$ tolerance ellipse (upper pole of the target) were categorized as "dehydrated" and short vectors outside the
$75 \%$ tolerance ellipse (lower pole of the target) as "hyperhydrated", while the remainder were "normohydrated". BNP was considered as a continuous variable and also divided into tertiles. Oxygenation index $\left(\mathrm{O}_{2} \mathrm{I}\right)$ was calculated as the mean airway pressure divided by the ratio of $\mathrm{PO}_{2} / \mathrm{FiO}_{2}$ and multiplied by 100 [4], and the result was then divided into tertiles.

Using least-squares regression, measures of CVP over time were used to estimate slope, and $\triangle \mathrm{CVP}$ was categorized as "falling" (<-2 cm/24h), "rising" ( $>2 \mathrm{~cm} / 24 \mathrm{~h})$, or "stable" ( $\triangle$ CVP from -2 to $2 \mathrm{~cm} / 24 \mathrm{~h}$ ). BIVA change over time was estimated as the slope of $\mathrm{R} / \mathrm{H}$ by time, and $>30 \mathrm{ohm} / \mathrm{m} /$ day was categorized as "falling ECF"; $<-30 \mathrm{ohm} / \mathrm{m} /$ day was "rising ECF", and values within $\pm 30 \mathrm{ohm} / \mathrm{m} /$ day were considered "stable ECF". Absolute values of BNP were used to estimate slope of BNP, and these slopes were divided into tertiles. Slope of $\mathrm{O}_{2} \mathrm{I}$ was grouped into tertiles. In the event that a patient was extubated, the last available mean airway pressure was carried forward, and the most recent arterial blood gas values were used to estimate the $\mathrm{O}_{2} \mathrm{I}$ for the purpose of calculating the slope.

The agreement between slopes of CVP, BIVA, and BNP against $\mathrm{O}_{2} \mathrm{I}$ were estimated using Kappa statistics. Univariate correlations were assessed at baseline for $\mathrm{O}_{2} \mathrm{I}, \mathrm{CVP}, \mathrm{BIVA}$, and BNP. Slopes of change were also assessed between these variables and hemodynamic parameters, fluid balance, and other clinical parameters such as hematocrit and creatinine. Multiple linear regression was performed with $\mathrm{O}_{2} \mathrm{I}$ and $\Delta \mathrm{O}_{2} \mathrm{I}$ as continuous dependent variables.

\section{Results}

Thirty-four patients were enrolled in the study, 22 (64.7\%) were male, and the most common admitting diagnosis was trauma, in 12 (35.3\%). Remaining baseline characteristics are presented in Table 1 . The majority of patients were not on pressors or inotropes at baseline, and the mean noradrenaline dose was $0.007 \pm 0.004 \mu \mathrm{cg} / \mathrm{kg} / \mathrm{min}$; dopamine was $1.6 \pm 0.5 \mu \mathrm{cg} / \mathrm{kg} / \mathrm{min}$. In cross-sectional analysis at baseline, there was no relationship between tertiles of CVP, BIVA or BNP with $\mathrm{O}_{2} \mathrm{I}$; however, a weak correlation could be demonstrated between the continuous variables of CVP and $\mathrm{O}_{2} \mathrm{I}(R=0.39 ; P=.021)$ (see Figure 1$)$ and a weak negative correlation between CVP and BIVA $(R=-0.38 ; P=.025)$.

Twenty-six subjects had data available beyond the first study day to allow estimation of slopes of change of CVP, BIVA, BNP, and $\mathrm{O}_{2} \mathrm{I}$. For these subjects, mean slope of change of CVP was $0.03 \mathrm{mmHg} /$ day, slope of BIVA was $-6.1 \mathrm{ohm} / \mathrm{m} /$ day, slope of $\mathrm{BNP}$ was $60.3 \mathrm{pg} / \mathrm{mL} /$ day and slope of $\mathrm{O}_{2} \mathrm{I}$ was 0.12 per day.

Comparing tertiles of CVP, BIVA, and BNP slopes with the slope of $\mathrm{O}_{2} \mathrm{I}$ revealed modest agreement between BNP and $\mathrm{O}_{2} \mathrm{I}$ (kappa $=0.25 ; P=.067$ ) and no agreement between the other variables. The relationship between tertiles of $\mathrm{BNP}$ and $\mathrm{O}_{2} \mathrm{I}$ slope is depicted in Figure 2. Similarly, using Spearman correlation, the slope of BNP was weakly correlated with $\mathrm{O}_{2} \mathrm{I}(R=0.40 ; P=.044)$ as shown in Figure 3. In a regression model examining all of the baseline variables and slopes of CVP, BIVA, and BNP as potential 
TABLE 1: Baseline characteristics $(N=34)$.

\begin{tabular}{lcc}
\hline Clinical variable & Mean & Standard error \\
\hline Age (years) & 59.2 & 3.6 \\
SOFA $^{\mathrm{a}}$ score day 1 & 7.1 & 0.4 \\
CVP $\left(\mathrm{cm} \mathrm{H}_{2} \mathrm{O}\right)$ & 10.4 & 0.6 \\
Resistance by height & 262.3 & 14.4 \\
(ohm/m) & 451.1 & 119.3 \\
BNP (pg/mL) & 0.32 & 0.01 \\
Hematocrit & 1.36 & 0.22 \\
Creatinine (mg/dL) & 132.8 & 4.5 \\
Systolic BP (mmHg) & 58.1 & 2.0 \\
Diastolic BP (mmHg) & 82.3 & 3.5 \\
Heart Rate (beats per & & \\
minute) & 89.9 & 2.8 \\
Mean Arterial Pressure & & 0.5 \\
(mmHg) & 9.3 & 0.6 \\
PEEP (mmHg) & 14.4 & 18.8 \\
$\begin{array}{l}\text { Mean Airway Pressure } \\
\text { (mmHg) }\end{array}$ & 288.4 & 0.5 \\
P/F ratio & 5.8 & \\
Oxygenation index & &
\end{tabular}

${ }^{a}$ Sequential Organ Failure Assessment score.

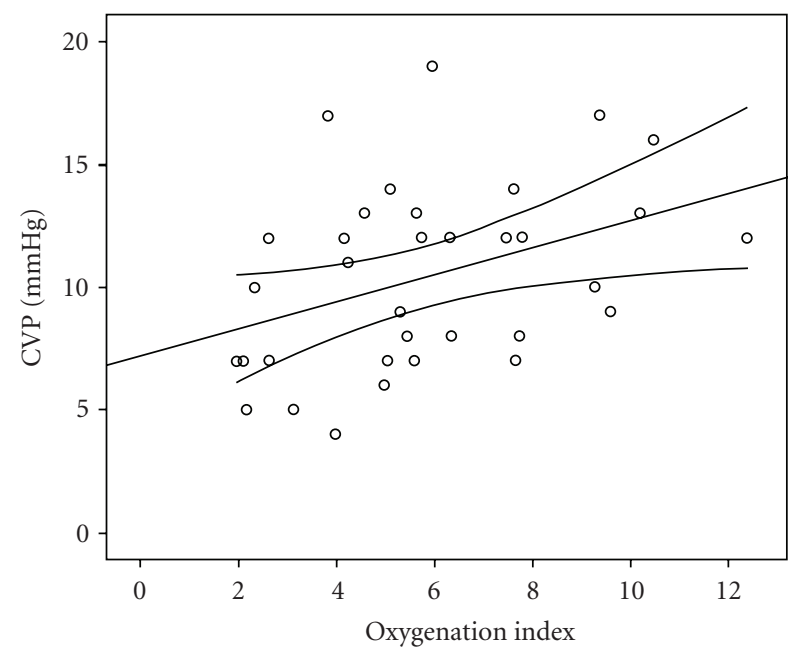

FIGURE 1: Correlation (with 95\% confidence intervals) between baseline CVP and Oxygenation Index $(R=0.39 ; P=.021)$.

variables, only BNP was significantly associated with $\mathrm{O}_{2} \mathrm{I}$, and this was strengthened by including CVP in the model. For each tertile increase in the slope of $\mathrm{BNP}, \mathrm{O}_{2} \mathrm{I}$ increased by 1.29 (95\% CI 0.18-2.41; $P=.025$ ).

\section{Conclusions}

This is the first study attempting to find the most appropriate combination of minimally invasive bedside tools for volume assessment in critically ill patients requiring mechanical ventilation. While none of the markers at baseline, individually

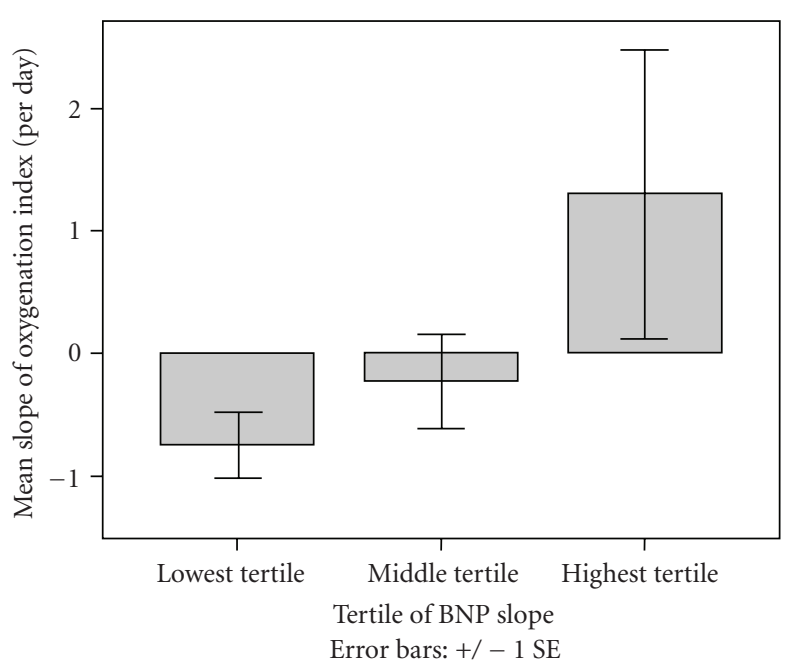

Figure 2: Mean slope of change of Oxygenation Index is shown categorized according to tertile of slope of change of BNP. Absolute difference between highest and lowest tertile $2.05 \pm 1.04, P=.067$ (ANOVA).

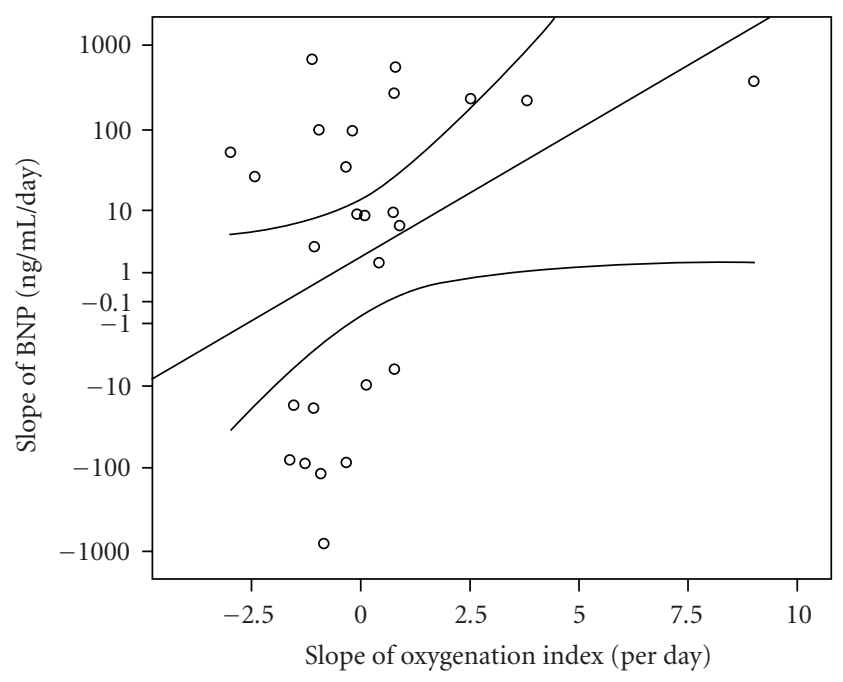

FIGURE 3: Correlation (with 95\% confidence intervals) between slope of BNP and slope of Oxygenation Index $(R=0.40 ; P=.044)$.

or in combination, were helpful in predicting those subjects whose oxygenation would improve or worsen, we identified that changes in BNP over time were correlated with important changes in $\mathrm{O}_{2} \mathrm{I}$. Furthermore, there was some indication that combining CVP and BNP in multivariable modeling further strengthened the latter variable's association with change in $\mathrm{O}_{2} \mathrm{I}$.

It is intuitive to think that measures of volume, be they measures of intravascular or extravascular (interstitial) volume, would be related to lung function and oxygenation. For instance, animal studies have shown that fluid balance can influence both the onset and resolution of severe "highpermeability" pulmonary edema $[12,13]$. Excess extravascular lung water is a feature of all types of pulmonary edema 
[14], and lower extravascular lung water correlates with fluid balance, decreased ventilator days, and ICU length of stay [15]. We chose CVP since local practice was such that most mechanically ventilated patients had central venous access appropriate for measurement of CVP. Furthermore, studies indicate a high level of agreement between clinical measures such as the external jugular pressure and the CVP [16]; hence, a ready estimate of CVP would be available in all subjects. We chose BIVA for its ease of use and noninvasive nature and its ability to provide an estimate of extravascular water. BNP was chosen for its ability to respond to myocardial stretch [17] and its utility in previous studies as a predictor of outcome $[9,10]$.

We did not find the addition of measures of BIVA to help in the fluid assessment of our cohort of patients. Previously, Piccoli and colleagues [6] demonstrated a modest degree of inverse correlation between CVP and impedance vector components, though this was stronger in the group that had significantly elevated CVP and weaker in the group with lower CVP. These authors suggested that the combination of CVP and BIVA might be useful in the volume assessment and management of critically ill patients. We were unable to demonstrate any correlation between BIVA and CVP, nor was the combination predictive of oxygenation. The principle difference between our study and that of Piccoli is that the minority of patients in the latter study were receiving mechanical ventilation, while this was a requirement for eligibility in our study. This may have played a role since mechanical ventilation with positive end-expiratory pressure (PEEP) likely and systematically elevated the CVP and possibly weakened any potential relationship between CVP and BIVA. Moreover, all the patients in our study had relatively high values of $\operatorname{PEEP}\left(8-10 \mathrm{cmH}_{2} \mathrm{O}\right)$.

We were able to demonstrate that change in BNP was associated with change in $\mathrm{O}_{2} \mathrm{I}$, and this relationship was strengthened modestly in multivariable regression by including slope of change of CVP. The choice of $\mathrm{O}_{2} \mathrm{I}$ as an outcome may be justly criticized as a surrogate, but the study population was not large enough for us to predict more clinically important outcomes such as lung injury scores, length of ICU stay, or days of mechanical ventilation, for example. However, $\mathrm{O}_{2} \mathrm{I}$ has been shown to be strongly associated with these more important outcomes in the Acute Respiratory Distress Syndrome (ARDS) clinical trials network study [4]. Another limitation of the study is that the methods used to assess volume were not compared against other methods such as echocardiography, ultrasound of the inferior vena cava, pulse pressure variation, or strove volume variation. This is a fair criticism; however, the study presented is the first in a series of pilot endeavours, the intent of which is to examine varying combinations of volume assessment. Additional studies utilizing the FloTrac Sensor and Vigileo Monitor (Edwards Lifesciences, S.A., Saint-Prex Switzerland) to measure stroke volume variation in critically ill patients in our institution are underway.

A larger study would have allowed us to explore more extreme values with greater confidence. For instance, inspection of the figures reveals greater variability at the extremes, and a larger sample size would have allowed a more sophisticated analysis of the relationships to see if nonlinear modeling would have provided a tighter fit with the data.

An additional limitation is the patient population, in whom the predominant admitting diagnosis was trauma (approximately one third). While the remainder had a variety of conditions including respiratory failure, decreased level of consciousness, sepsis, and intracranial hemorrhage or stroke; generalizability to the critical care population as a whole is difficult. Another potential limitation relates to our choice of waiting for 48-72 hours to enroll patients, during which time they could have stabilized to a point that may have dampened the strength of the relationships we observed.

Our results are consistent with the recent work of Levitt and colleagues [18], who carried out a similar prospective cohort study of critically ill patients and compared various measures of volume status. As in their study, we found no relationship between CVP and BNP over time. However, our study differs in that we examined the combination of these parameters in multivariable modeling and found them to provide complementary information in predicting improvements in oxygenation.

In summary, bedside measures of volume status, CVP and BIVA, were unhelpful alone in predicting favorable changes in $\mathrm{O}_{2} \mathrm{I}$, while changes in BNP over time did correlate with changes in $\mathrm{O}_{2} \mathrm{I}$. There was some indication that combining CVP and BNP improved the ability to predict change in $\mathrm{O}_{2} \mathrm{I}$. Whether or not interventions to optimize both CVP and BNP will result in improved outcomes in ventilated, critically ill patients will require further prospective study.

\section{Acknowledgments}

The authors are deeply grateful to the physicians and nurses of the San Bortolo Hospital Intensive Care Unit for their assistance in identifying eligible subjects for the study, and for capturing the important hemodynamic and clinical parameters necessary for the analysis.

\section{References}

[1] M. Hadian and M. R. Pinsky, "Functional hemodynamic monitoring," Current Opinion in Critical Care, vol. 13, no. 3, pp. 318-323, 2007.

[2] E. Rivers, B. Nguyen, S. Havstad et al., "Early goal-directed therapy in the treatment of severe sepsis and septic shock," The New England Journal of Medicine, vol. 345, no. 19, pp. 13681377, 2001.

[3] S. M. Lin, C. D. Huang, H. C. Lin, C. Y. Liu, C. H. Wang, and H. P. Kuo, "A modified goal-directed protocol improves clinical outcomes in intensive care unit patients with septic shock: a randomized controlled trial," Shock, vol. 26, no. 6, pp. 551-557, 2006.

[4] H. P. Wiedemann, A. P. Wheeler, G. R. Bernard et al., "Comparison of two fluid-management strategies in acute lung injury," New England Journal of Medicine, vol. 354, no. 24, pp. 2564-2575, 2006.

[5] A. P. Wheeler, G. R. Bernard, B. T. Thompson et al., "Pulmonary-artery versus central venous catheter to guide treatment of acute lung injury," New England Journal of Medicine, vol. 354, no. 21, pp. 2213-2224, 2006. 
[6] A. Piccoli, G. Pittoni, E. Facco, E. Favaro, and L. Pillon, "Relationship between central venous pressure and bioimpedance vector analysis in critically ill patients," Critical Care Medicine, vol. 28, no. 1, pp. 132-137, 2000.

[7] A. Piccoli, S. Nigrelli, A. Caberlotto et al., "Bivariate normal values of the bioelectrical impedance vector in adult and elderly populations," American Journal of Clinical Nutrition, vol. 61, no. 2, pp. 269-270, 1995.

[8] P. A. McCullough, P. Duc, T. Omland et al., "B-type natriuretic peptide and renal function in the diagnosis of heart failure: an analysis from the Breathing Not Properly Multinational Study," American Journal of Kidney Diseases, vol. 41, no. 3, pp. 571-579, 2003.

[9] M. Varpula, K. Pulkki, S. Karlsson, E. Ruokonen, and V. Pettilä, "Predictive value of N-terminal pro-brain natriuretic peptide in severe sepsis and septic shock," Critical Care Medicine, vol. 35, no. 5, pp. 1277-1283, 2007.

[10] B. Meyer, M. Huelsmann, P. Wexberg et al., "N-terminal pro-B-type natriuretic peptide is an independent predictor of outcome in an unselected cohort of critically ill patients," Critical Care Medicine, vol. 35, no. 10, pp. 2268-2273, 2007.

[11] R. F. Kushner, "Bioelectrical impedance analysis: a review of principles and applications," Journal of the American College of Nutrition, vol. 11, no. 2, pp. 199-209, 1992.

[12] C. Mitaka, Y. Hirata, K. Habuka et al., "Atrial natriuretic peptide improves pulmonary gas exchange by reducing extravascular lung water in canine model with oleic acidinduced pulmonary edema," Critical Care Medicine, vol. 30, no. 7, pp. 1570-1575, 2002.

[13] E. D. Sivak, J. Tita, and G. Meden, "Effects of furosemide versus isolated ultrafiltration on extravascular lung water in oleic acid-induced pulmonary edema," Critical Care Medicine, vol. 14 , no. 1 , pp. 48-51, 1986.

[14] G. M. Rocker, A. G. Morgan, and D. J. Shale, "Pulmonary oedema and renal failure," Nephrology Dialysis Transplantation, vol. 3, no. 3, pp. 244-246, 1988.

[15] J. P. Mitchell, D. Schuller, F. S. Calandrino, and D. P. Schuster, "Improved outcome based on fluid management in critically ill patients requiring pulmonary artery catheterization," American Review of Respiratory Disease, vol. 145, no. 5, pp. 990-998, 1992.

[16] A. G. Vinayak, J. Levitt, B. Gehlbach, A. S. Pohlman, J. B. Hall, and J. P. Kress, "Usefulness of the external jugular vein examination in detecting abnormal central venous pressure in critically ill patients," Archives of Internal Medicine, vol. 166, no. 19, pp. 2132-2137, 2006.

[17] M. Suresh and K. Farrington, "Natriuretic peptides and the dialysis patient," Seminars in Dialysis, vol. 18, no. 5, pp. 409419, 2005.

[18] J. E. Levitt, A. G. Vinayak, B. K. Gehlbach et al., "Diagnostic utility of B-type natriuretic peptide in critically ill patients with pulmonary edema: a prospective cohort study," Critical Care, vol. 12, no. 1, article no. R3, 2008. 


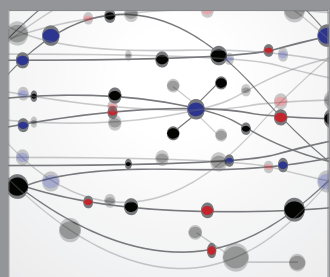

The Scientific World Journal
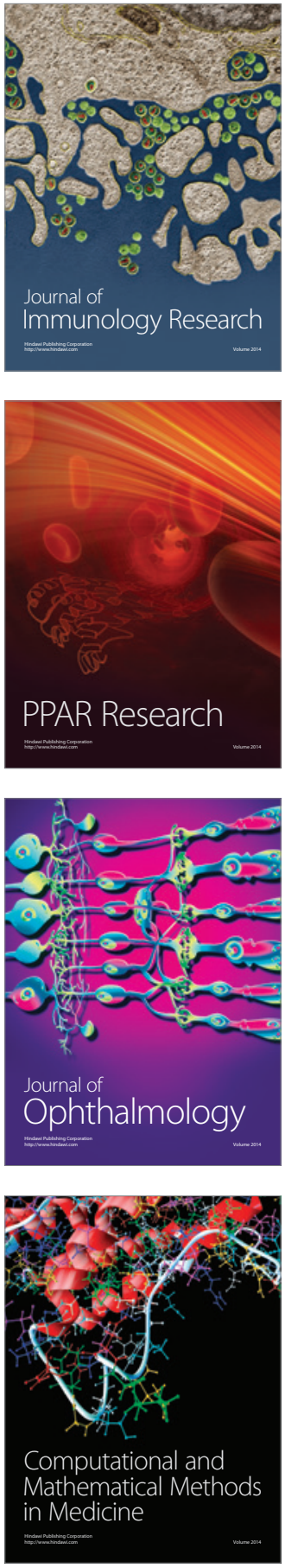

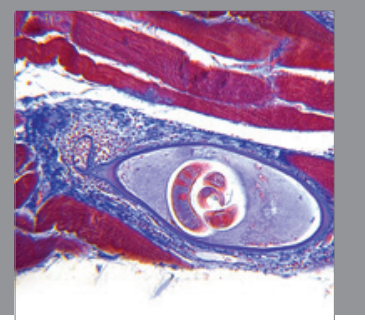

Gastroenterology

Research and Practice
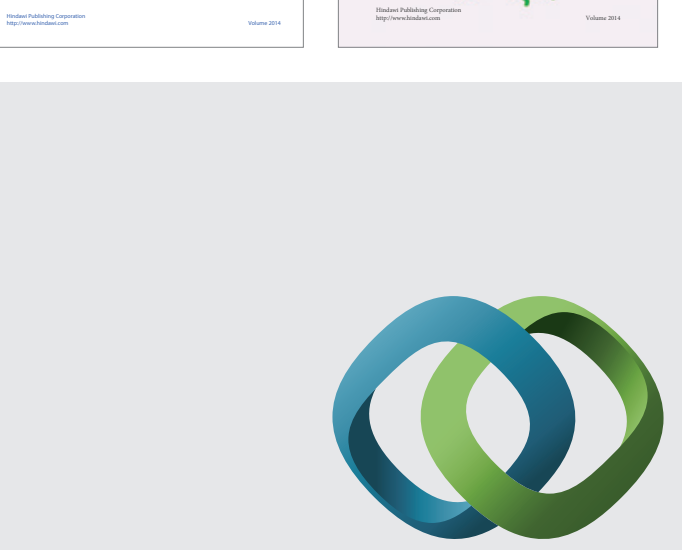

\section{Hindawi}

Submit your manuscripts at

http://www.hindawi.com
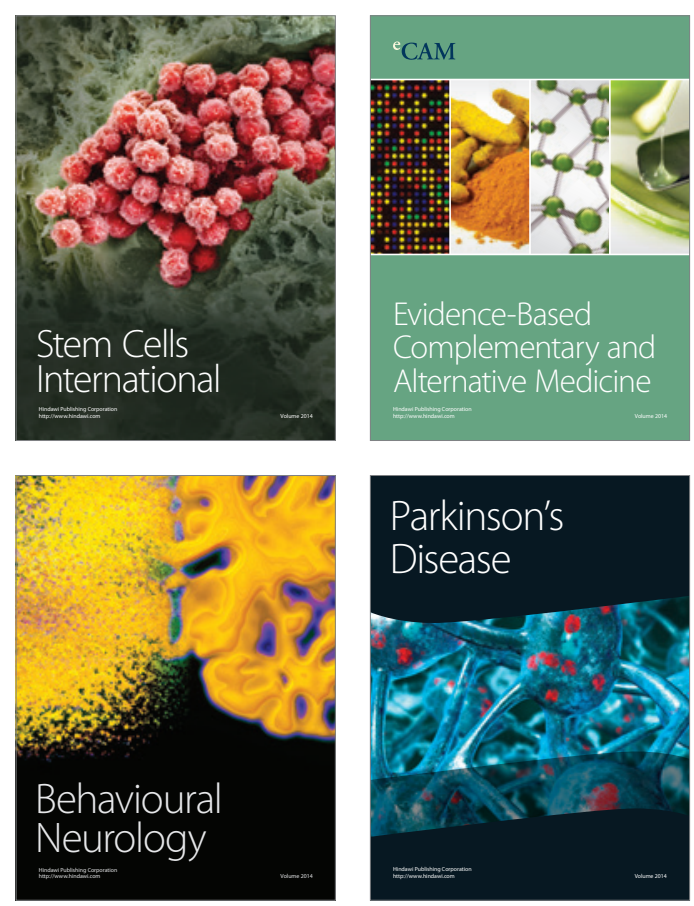

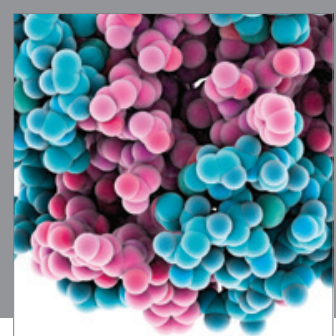

Journal of
Diabetes Research

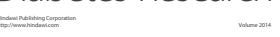

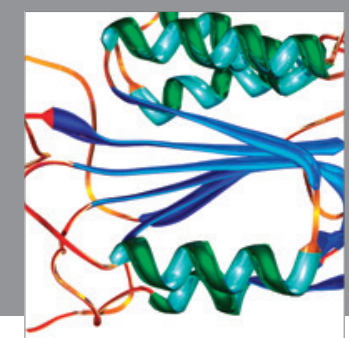

Disease Markers
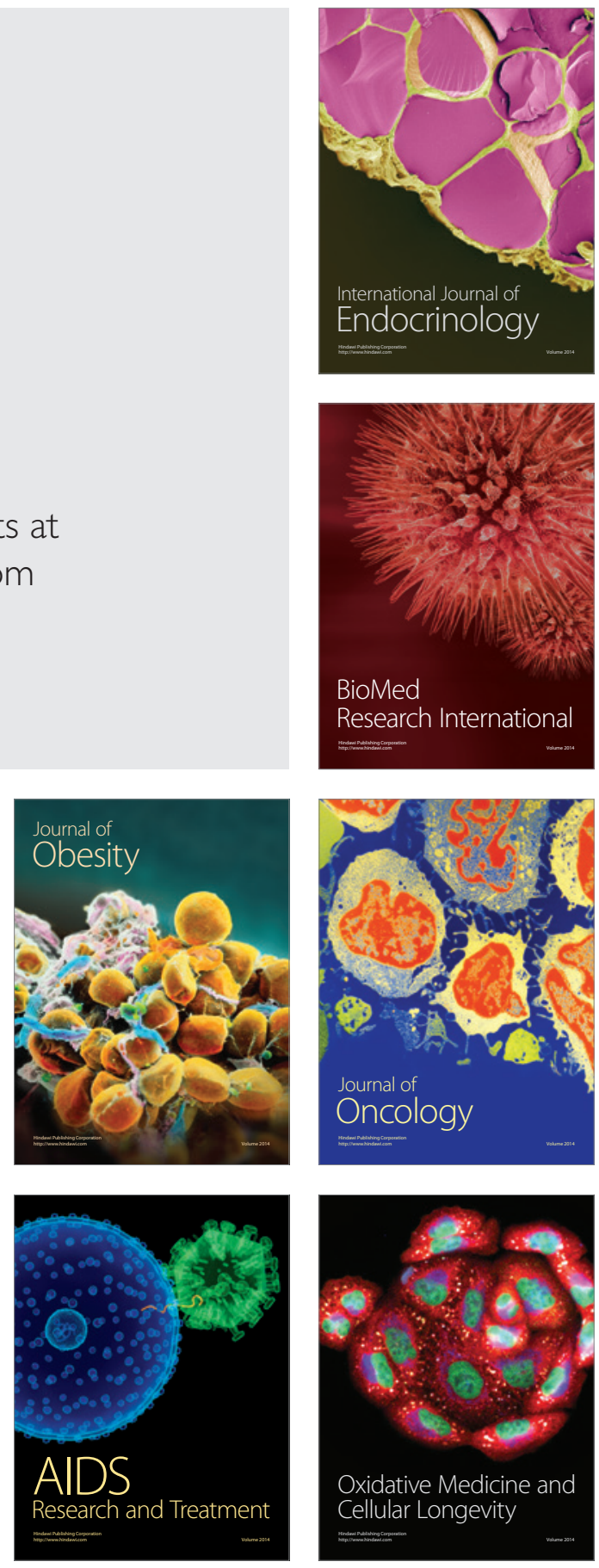